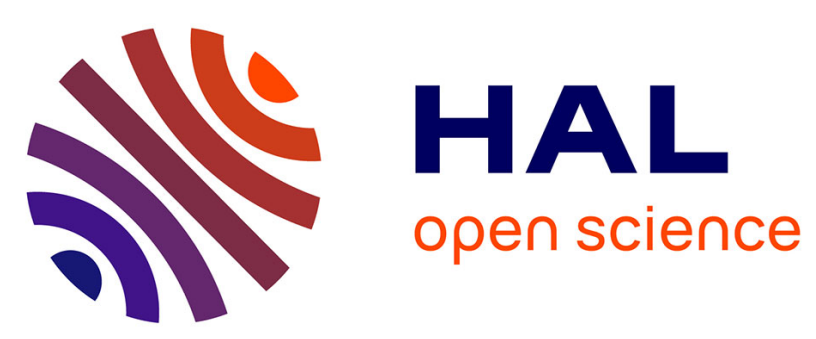

\title{
Constraints on Equation of State for Cavitating Flows with Thermodynamic Effects
}

Eric Goncalvès da Silva, Regiane. Fortes Patella

\section{To cite this version:}

Eric Goncalvès da Silva, Regiane . Fortes Patella. Constraints on Equation of State for Cavitating Flows with Thermodynamic Effects. Applied Mathematics and Computation, 2011, 217, pp.5095-5102. 10.1016/j.amc.2010.07.056 . hal-00530263

\section{HAL Id: hal-00530263 https://hal.science/hal-00530263}

Submitted on 28 Oct 2010

HAL is a multi-disciplinary open access archive for the deposit and dissemination of scientific research documents, whether they are published or not. The documents may come from teaching and research institutions in France or abroad, or from public or private research centers.
L'archive ouverte pluridisciplinaire HAL, est destinée au dépôt et à la diffusion de documents scientifiques de niveau recherche, publiés ou non, émanant des établissements d'enseignement et de recherche français ou étrangers, des laboratoires publics ou privés. 


\title{
Constraints on Equation of State for Cavitating Flows with Thermodynamic Effects
}

\author{
Eric Goncalvès *, Regiane Fortes Patella \\ LEGI, Grenoble-INP, 1025 rue de la Piscine, 38400 St Martin d'Heres, France
}

\begin{abstract}
Thermodynamic effects play an important role in the cavitation dynamics of cryogenics fluids. Such flows are characterized by strong variations in fluid properties with the temperature. A compressible, multiphase, one-fluid solver was developed to study and to predict thermodynamic effects in cavitating flows. To close the system, a cavitation model is proposed to capture metastable behaviours of fluids and non isothermal thermodynamic path. The thermodynamical consistency based on entropy conditions and the evolution of the mixture speed of sound are investigated. These constraints are applied to other models. The considered working fluid is the refrigerant R-114.
\end{abstract}

Key words: Cavitation, Homogeneous Model, Thermodynamic Effect, Equation of State, Entropy Conditions, Mixture Sound Speed

\section{Notations}

$c$

speed of sound

* Corresponding author.

Email address: Eric.Goncalves@legi.grenoble-inp.fr (Eric Goncalvès ). 


$\begin{array}{ll}e & \text { specific internal energy } \\ g & \text { specific Gibbs free enthalpy } \\ h & \text { specific enthalpy } \\ q & \text { reference energy of stiffened gas } \\ s & \text { specific entropy } \\ C_{p}, C_{v} & \text { thermal capacities } \\ M & \text { Mach number } \\ P & \text { static pressure } \\ P_{v a p} & \text { vapour pressure } \\ P_{\infty} & \text { reference pressure of stiffened gas } \\ T & \text { static temperature } \\ T_{r e f} & \text { reference temperature } \\ \alpha & \text { void ratio } \\ \gamma & \text { heat capacity ratio } \\ \rho & \text { density } \\ \mu & \text { dynamic viscosity }\end{array}$

Subscripts and superscripts
$L \quad$ liquid value
$V \quad$ vapour value

\section{Introduction}

The simulation and the prediction of cavitation in cryogenic fluids is of critical importance for the efficient design and performance of turbopumps in rocket propulsion systems. With thermosensible fluids, thermal effects and strong 
variations in fluid properties are observed, which alter the cavity characteristics. For such fluids, the liquid-vapour density ratio is lower than in cold water and consequently more liquid mass has to vaporize to sustain a cavity. Therefore evaporative cooling effects are more pronounced and the temperature of the liquid in the immediate vicinity of the liquid-vapour interface is depressed below the free-stream temperature. Because of the strong variation of thermodynamic properties (mainly the vapour pressure), the temperature depression, negligible in water, is quite substantial. The local cooling effect delays the cavitation phenomenon and reduces the local vapour pressure of the fluid, which leads to a lower observed cavity pressure. Typical temperature depressions in cryogenic fluids are one to two Kelvin, this can correspond to changes in vapour pressure of a factor of two.

Various numerical models, within the framework of the continuum modelling method, have been developed to investigate thermodynamic effects in cavitating flows. This method makes no attempt to track the liquid and vapour interface but treats the flow as two-phases with an averaged mixture density, which continuously varies between the liquid and vapour extremes. In its implementation, there are different approaches according to the assumptions made: equilibrium models versus non-equilibrium models, homogeneous models versus two-fluid models.

The two-fluid approach assumes that both phases co-exist at every point in the flow field and each phase is governed by its own set of conservation laws. These models can take into account the physical details occurring in the cavitation phenomenon such as mass exchange, thermal transfer and surface tension. However, transfer terms have to be known; such quantities are usually very difficult to obtain.

The one-fluid method treats the cavitating flows as a mixture of two fluids behaving as one. These models assume a no-slip condition between phases. 
With the assumption of local thermodynamic equilibrium (local temperature, pressure and free Gibbs enthalpy equality between phases), they constitute the homogeneous equilibrium models (HEM). An equation of state (EOS) is necessary to close the system. Different closure relations that link the pressure to the temperature and the density have been proposed [1-3]. Small non-equilibrium effects can be introduced in the EOS compared to an isothermal thermodynamic path. When non-equilibrium effect becomes important, additional equations are needed for an accurate prediction.

A four-equation model, very popular to simulate cavitating flows in cold water, has been adapted to cryogenic applications [4-7]. It is obtained by adding a mass equation for the vapour or liquid density including a cavitation source term. The main difficulty is related to the formulation of the source term and the tunable parameters involved for the vaporization and condensation processes (different sets of parameters are presented in [6]).

The present work is part of a research aimed at developing a numerical tool devoted to cavitating flows. Particular emphasis is placed on the study of thermodynamical properties of models and equations of state. In previous works $[8,9]$, an in-house finite-volume code solving the Reynolds-Averaged Navier-Stokes (RANS) compressible equations was developed with an homogeneous approach. Different constraints on the EOS was introduced. This paper complements the previous analysis. Physical constraints (entropy conditions and evolution of the mixture sound speed) are proposed, for the inviscid Euler equations, to ensure the thermodynamical coherence of models. These constraints make possible the thermodynamical analysis of different cavitation models. Given this methodology, we evaluate several models: a modified barotropic EOS, a mixture of stiffened gas, and a four-equation model. The chosen working fluid is the freon R-114 $\left(\mathrm{C}_{2} \mathrm{Cl}_{2} F_{4}\right)$.

The paper is organized as follows: the homogeneous inviscid system is pre- 
sented, including the pure phases equation of state. This is followed by the constraints on EOS and the study of different cavitation models.

\section{The one-fluid Euler equations}

The homogeneous model assumes strong coupling of the phases and moving at the same velocity components. The phases are assumed in close proximity to each other so that heat transfer would occur instantaneously maintaining the phases in thermal equilibrium. Furthermore, the disperse phase behaves quasi-statically to change in pressure and the mixture are assumed in constant pressure. Therefore the phases share the same temperature $T$ and the same pressure $P$.

The evolution of the two-phase flow can be described by Euler equations that employ the representative flow properties as unknowns just as a single-phase problem. The mixture density $\rho$ is defined by:

$$
\rho=\alpha \rho_{V}+(1-\alpha) \rho_{L}
$$

where $\rho_{L}$ and $\rho_{V}$ are respectively the liquid and vapour densities. The void ratio $\alpha$ characterizes the volume of vapour in each cell: $\alpha=1$ means that the cell is completely filled by vapour; inversely, a complete liquid cell is represented by $\alpha=0$. Liquid and vapour phases are characterized by their thermodynamic properties. On each cell, the unknowns are calculated by averaging them by the volume occupied.

In order to simplify the formulation, we present below the one-dimensional equations, expressed in conservative variables $w=(\rho, \rho u, \rho E)$ :

$$
\frac{\partial \rho}{\partial t}+\frac{\partial \rho u}{\partial x}=0
$$




$$
\begin{aligned}
\frac{\partial(\rho u)}{\partial t}+\frac{\partial\left(\rho u^{2}+P\right)}{\partial x} & =0 \\
\frac{\partial(\rho E)}{\partial t}+\frac{\partial(\rho u H)}{\partial x} & =0
\end{aligned}
$$

where $E=e+u^{2} / 2$ denotes the total energy and $H=h+u^{2} / 2$ the total enthalpy; $e$ the internal energy, $h$ the enthalpy, which are related to the void ratio and the liquid and vapour values:

$$
\begin{aligned}
& \rho e=\alpha \rho_{V} e_{V}+(1-\alpha) \rho_{L} e_{L} \\
& \rho h=\alpha \rho_{V} h_{V}+(1-\alpha) \rho_{L} h_{L}
\end{aligned}
$$

To close the system, an equation of state (EOS) is necessary to link the pressure and the temperature to the internal energy and the density. The difficulty with this homogeneous approach is to specify an EOS that covers all possible fluid states: pure liquid, two-phase mixture and pure gas. For the pure phases, we considered the convex stiffened gas EOS:

$$
\begin{aligned}
P(\rho, e) & =(\gamma-1) \rho(e-q)-\gamma P_{\infty} \\
P(\rho, T) & =\rho(\gamma-1) C_{v} T-P_{\infty} \\
T(\rho, h) & =\frac{h-q}{C_{p}}
\end{aligned}
$$

where $q$ is the energy of the fluid at a given reference state and $P_{\infty}$ a constant reference pressure.

\section{Constraints on models}

\subsection{Entropy conditions}

A suitable mixture EOS has to provide stable solutions, which respect the second principle of thermodynamics. Given a two-phase mixture with an entropy, a thermodynamic state is said to be stable if it is an absolute maximum 
of the entropy and it is metastable if it is a relative maximum of the entropy. This induces the fact that the entropy $s$ of a given state must always ensure the following criterion: $d^{2} s<0$ (condition of concave entropy). Moreover, the concavity of $s$ is equivalent to the convexity of $e$ (i.e. the Hessian matrix of internal energy has to be positive definite).

The convexity of internal energy is therefore necessary to ensure thermodynamic stability and to respect the entropy growth criterion. An equivalent condition of convexity was formulated for two-phase flows with phase transition, which implies that these three relations have to be verified $[10,11]$ :

$$
\begin{aligned}
\lambda=\frac{\rho}{P}\left(\frac{\partial P}{\partial \rho}\right)_{s} \geq 0 & \text { and } & g & =\frac{P}{\rho T^{2}}\left(\frac{\partial T}{\partial s}\right)_{\rho} \geq 0 \\
\lambda g-\Gamma^{2} \geq 0 & \text { with } & \Gamma & =\frac{\rho}{T}\left(\frac{\partial T}{\partial \rho}\right)_{s}
\end{aligned}
$$

\subsection{Mixture speed of sound conditions}

Without mass transfer effects, the propagation of acoustic waves follows the Wallis speed of sound [12]. This speed $c_{\text {wallis }}$ is expressed as a weighted harmonic mean of speeds of sound of each phase:

$$
\frac{1}{\rho c_{\text {wallis }}^{2}}=\frac{\alpha}{\rho_{V} c_{V}^{2}}+\frac{1-\alpha}{\rho_{L} c_{L}^{2}}
$$

When exchanges of mass and heat between phases are involved, the sound speed decreases to the thermodynamic equilibrium one [13]. This limit speed is evaluated with the assumption of local thermodynamic equilibrium: equalities of pressure, temperature and free enthalpy $g=h-T s$ between phases. The complete formulation of this model is given in [14]. The expression of the speed of sound $c_{e q}$ is (see Appendix): 


$$
\begin{aligned}
c_{e q}^{2} & =\frac{h-\left(\frac{\partial \rho e}{\partial \rho}\right)_{P}}{\left(\frac{\partial \rho e}{\partial P}\right)_{\rho}} \\
\left(\frac{\partial \rho e}{\partial \rho}\right)_{P} & =\frac{\rho_{L} h_{L}-\rho_{V} h_{V}}{\rho_{L}-\rho_{V}} \\
\left(\frac{\partial \rho e}{\partial P}\right)_{\rho} & =\frac{1}{\gamma-1}-\frac{\rho_{L} h_{L}-\rho_{V} h_{V}}{\rho_{L}-\rho_{V}} \frac{1}{T} \\
& \times\left[\alpha\left(\frac{1}{C v_{V}\left(\gamma_{V}-1\right)}-\rho_{V} \frac{d T}{d P_{\text {vap }}}\right)+(1-\alpha)\left(\frac{1}{C v_{L}\left(\gamma_{L}-1\right)}-\rho_{L} \frac{d T}{d P_{\text {vap }}}\right)\right]
\end{aligned}
$$

Therefore, to ensure a thermodynamic coherence, the mixture speed of sound given by models has to vary between the Wallis and the equilibrium ones:

$$
c_{e q} \leq c<c_{\text {wallis }}
$$

\section{Study of a modified barotropic law}

In the mixture, the cavitation model is based on a barotropic law $[15,8,9]$. This law is modified by introducing thermal variations. When the pressure becomes smaller than the quantity $P_{v a p}(T)+\Delta P$ and greater than $P_{v a p}(T)-\Delta P$, we have the relation:

$$
P(\alpha, T)=P_{v a p}(T)+\left(\frac{\rho_{L}\left(T_{r e f}\right)-\rho_{V}\left(T_{r e f}\right)}{2}\right) c_{b a r o}^{2} \operatorname{Arcsin}(1-2 \alpha)
$$

where $\Delta P$ represents the pressure width of the law and, for a void ratio $\alpha=0.5$, the pressure is equal to the vaporization pressure $P_{v a p}(T)$. The quantity $c_{b a r o}$ is an adjustable parameter of the model which has the dimension of a velocity. $\rho_{L}\left(T_{r e f}\right)$ and $\rho_{V}\left(T_{r e f}\right)$ are saturation values of liquid and vapour densities, evaluated at the reference temperature $T_{r e f}$, respectively. 
We assume that the vaporization pressure varies linearly with the temperature:

$$
P_{v a p}(T)=P_{v a p}\left(T_{r e f}\right)+\frac{d P_{v a p}}{d T}\left(T-T_{r e f}\right)
$$

For an equilibrium two-phase flow, the pressure depends only on the temperature, derivatives of $P$ and $T$ with respect to the density are zero. With this EOS, these quantities are no more equal to zero, leading to a weak nonequilibrium effect. The constant quantity $d P_{\text {vap }} / d T$, determined with a thermodynamic table, is fixed to $6000 \mathrm{~Pa} / \mathrm{K}$ for freon $\mathrm{R}-114$ at $T_{r e f}=293 \mathrm{~K}$.

To respect the continuity of the pressure between the liquid and the mixture (when $P=P_{v a p}+\Delta P$ ), we have the relation:

$$
\Delta P=\frac{\rho_{L}-\rho_{V}}{2} c_{\text {baro }}^{2} \frac{\pi}{2}=\rho_{L}\left(\gamma_{L}-1\right) C_{v_{L}} T_{r e f}-P_{\infty}^{L}-P_{\text {vap }}\left(T_{\text {ref }}\right)
$$

This relation sets $c_{b a r o}$ for given values of saturation conditions. The continuity between the pure vapour and the mixture is not considered because, in our applications, pure gas is never reached. Numerical results obtained on a Venturi geometry are presented in [9].

\subsection{Convexity conditions}

With our choice of parameters for the model (values are given in Table 1), the first two conditions are well respected. Moreover, the third convexity relation can be expressed as:

$$
\begin{array}{r}
K_{2}=c_{T}^{2}+\frac{T \frac{d P_{v a p}}{d T}}{2 \rho\left(K_{1}-1\right)} \\
K_{2}-\sqrt{K_{2}^{2}-c_{T}^{4}} \leq c^{2} \leq K_{2}+\sqrt{K_{2}^{2}-c_{T}^{4}}
\end{array}
$$


This inequality is respected for void ratio values depending on the choice of the parameter $c_{\text {baro }}$. For example, for the freon R-114, if $c_{\text {baro }}=1.48 \mathrm{~m} / \mathrm{s}$, the convexity is verified for void ratio values smaller than 0.8 . The convexity domains are given in Table 2 for different values of the parameter $c_{b a r o}$.

\subsection{Evolution of the mixture speed of sound}

The speed of sound can be expressed as [9]:

$$
\begin{aligned}
\rho C_{p} & =\alpha \rho_{V} C_{p_{V}}+(1-\alpha) \rho_{L} C_{p_{L}} \\
\left(K_{1}-1\right) c^{2} & =\frac{\rho_{V} \rho_{L}}{\rho\left(\rho_{L}-\rho_{V}\right)}\left(h_{V}-h_{L}\right)-\rho C_{p}\left(\frac{\partial T}{\partial \rho}\right)_{P} \\
\text { with }\left(\frac{\partial \rho}{\partial T}\right)_{P} & =-\frac{d P_{\text {vap }}}{d T} \frac{1}{c_{\text {baro }}^{2}} \cos \left(\frac{P-P_{\text {vap }}(T)}{0.5\left(\rho_{L}-\rho_{V}\right) c_{\text {baro }}^{2}}\right) \\
\text { and } K_{1} & =\frac{\rho C_{p}}{\frac{d P_{\text {vap }}}{d T}}
\end{aligned}
$$

Introducing the isothermal speed of sound $c_{T}$ (i.e. when $\left.d P_{v a p} / d T=0\right)$ :

$$
c_{T}^{2}=\left(\frac{\partial P}{\partial \rho}\right)_{s}=\left(\frac{\partial P}{\partial \rho}\right)_{T}=\frac{c_{\text {baro }}^{2}}{2 \sqrt{\alpha(1-\alpha)}}
$$

The speed of sound in the mixture can be written as:

$$
\begin{aligned}
\left(\frac{\partial T}{\partial \rho}\right)_{P} & =-c_{T}^{2} \frac{d T}{d P_{v a p}} \\
c^{2} & =\frac{\frac{\rho_{V} \rho_{L}}{\rho\left(\rho_{L}-\rho_{V}\right)}\left(h_{V}-h_{L}\right) \frac{d P_{v a p}}{d T}+\rho C_{p} c_{T}^{2}}{\rho C_{p}-\frac{d P_{v a p}}{d T}}
\end{aligned}
$$

With our choice of parameters for the model (values are given in Table 1), $c^{2}$ is always positive, therefore the inviscid system is hyperbolic.

Figures 1 and 2 compare the evolution of the speed of sound in a mixture 
of freon R-114, in function of the void ratio, for the isothermal and modified barotropic EOS, the Wallis formulation and the equilibrium model. To compare the different speeds, the temperature is assumed to be constant equal to 293K and saturation values are used for $\rho_{L}, \rho_{V}, h_{L}$ and $h_{V}$.

For the isothermal barotropic law, we can see that the mixture speed of sound is smaller than the equilibrium one. This model does not respect the thermodynamical constraint on the mixture speed of sound.

On the contrary, for the modified barotropic EOS, the mixture speed of sound is well limited by the Wallis and the equilibrium ones. Moreover, the speed of sound obtained is not symmetric. The minimum value (around $2.5 \mathrm{~m} / \mathrm{s}$ ) is reached for void ratio close to zero.

\section{$5 \quad$ Study of two other models}

The proposed constraints make possible the analysis of the thermodynamical coherence of different cavitation models. We present the study of two other models: a mixture of stiffened gas and a four-equation model.

\subsection{Mixture of stiffened gas}

This model is described in [9], in which the convexity conditions are already studied. The mixture speed of sound can be expressed as a function of the enthalpy of each phase:

$$
\rho c^{2}=\rho\left(\frac{\partial P}{\partial \rho}\right)_{s}=(\gamma-1)\left[\frac{\rho_{V} \rho_{L}}{\left(\rho_{L}-\rho_{V}\right)}\left(h_{V}-h_{L}\right)\right]
$$

Figure 3 compares the evolution of the speed of sound in the mixture as a function of the void ratio. The temperature is assumed to be constant equal to $293 \mathrm{~K}$ and saturation values are used for $\rho_{L}, \rho_{V}, h_{L}$ and $h_{V}$. We can observe 
the good behaviour of the mixture speed of sound, which verifies the inequality $(15)$.

\subsection{Four-equation model}

This model is composed by three conservation laws for the mixture and a supplementary equation for the mass of one pure phase. To simplify the formulation, we present only the one-dimensional inviscid equations.

$$
\begin{aligned}
\frac{\partial \rho}{\partial t}+\frac{\partial \rho u}{\partial x} & =0 \\
\text { or } \quad \frac{\partial \alpha \rho_{V}}{\partial t}+\frac{\partial \alpha \rho_{V} u}{\partial x} & =\dot{m} \\
\frac{\partial(1-\alpha) \rho_{L}}{\partial t}+\frac{\partial(1-\alpha) \rho_{L} u}{\partial x} & =-\dot{m} \\
\frac{\partial \rho u}{\partial t}+\frac{\partial\left(\rho u^{2}+P\right)}{\partial x} & =0 \\
\frac{\partial \rho E}{\partial t}+\frac{\partial(\rho u H)}{\partial x} & =0
\end{aligned}
$$

where the mass transfer between phases $\dot{m}$ involves a vaporization and a condensation contribution. It can be expressed as [4]:

$$
\dot{m}=\dot{m}^{+}+\dot{m}^{-}=\frac{C^{-}}{\tau} \rho_{V}(1-\alpha) \frac{\operatorname{Min}\left(0, P-P_{v a p}\right)}{0.5 \rho_{r e f} U_{r e f}^{2}}+\frac{C^{+}}{\tau} \rho_{V} \alpha(1-\alpha)^{2}(33)
$$

where $\tau$ is a characteristic time, and $C^{+}, C^{-}$are empirical constants.

With assumptions of mechanical and thermal equilibrium between pure phases (i.e. $P_{V}=P_{L}=P$ and $T_{L}=T_{V}=T$ ), a void ratio equation can be expressed (the demonstration for a five-equation model is given in [14]):

$$
\frac{\partial \alpha}{\partial t}+u \frac{\partial \alpha}{\partial x}=\underbrace{\left(\frac{\rho_{L} c_{L}^{2}-\rho_{V} c_{V}^{2}}{\frac{\rho_{L} c_{L}^{2}}{1-\alpha}+\frac{\rho_{V} c_{V}^{2}}{\alpha}}\right)}_{=K} \frac{\partial u}{\partial x}+\underbrace{\left(\frac{\frac{c_{V}^{2}}{\alpha}+\frac{c_{L}^{2}}{1-\alpha}}{\frac{\rho_{L} c_{L}^{2}}{1-\alpha}+\frac{\rho_{V} c_{V}^{2}}{\alpha}}\right)}_{=1 / \rho_{I} \text { the interfacial density }} \dot{m}
$$


The pressure equation is deduced:

$$
\frac{\partial P}{\partial t}+u \frac{\partial P}{\partial x}+\rho_{V} c_{V}^{2}\left(1+\frac{K}{\alpha}\right) \frac{\partial u}{\partial x}=\frac{c_{V}^{2}}{\alpha}\left(1-\frac{\rho_{V}}{\rho_{I}}\right) \dot{m}
$$

To compute the mixture speed of sound, the system is written with the primitive variable $(\alpha, P, u, e)$. We introduce the quantity $C=\rho_{V} c_{V}^{2}\left(1+\frac{K}{\alpha}\right)$.

$$
\frac{\partial}{\partial t}\left(\begin{array}{c}
\alpha \\
P \\
u \\
e
\end{array}\right)+\left[\begin{array}{cccc}
u & 0 & -K & 0 \\
0 & u & C & 0 \\
0 & 1 / \rho & u & 0 \\
0 & 0 & P / \rho & u
\end{array}\right] \frac{\partial}{\partial x}\left(\begin{array}{c}
\alpha \\
P \\
u \\
e
\end{array}\right)=\rho_{I} \dot{m}\left(\begin{array}{c}
\alpha \\
c_{V}^{2}\left(\rho_{I}-\rho_{V}\right) / \alpha \\
0 \\
0
\end{array}\right)
$$

The eigenvalues of the matrix of the system can be easily computed. The system is hyperbolic, eigenvalues are $(u, u, u-c, u+c)$, with the following expression for the mixture speed of sound:

$$
\rho c^{2}=C=\frac{\rho_{L} \rho_{V} c_{L}^{2} c_{V}^{2}}{\alpha \rho_{L} c_{L}^{2}+(1-\alpha) \rho_{V} c_{V}^{2}}=\rho c_{w a l l i s}^{2}
$$

This model reproduces propagation of acoustic disturbance at the Wallis speed of sound that is not thermodynamically coherent. To decrease the mixture speed of sound, the source term has to involve derivatives of variables.

\section{Conclusion}

A set of constraints has been proposed to study the thermodynamical consistency of cavitation models or mixture equations of state. These constraints involve the respect of the second principle of thermodynamic through the convexity conditions for the inviscid system. In addition, conditions on the mixture speed of sound have been presented: the speed of sound has to be 
limited by both Wallis and equilibrium velocities.

These constraints have been applied to different cavitation models. The condition on the mixture speed of sound appears to be discriminating. Both the isothermal barotropic law and the popular four-equation model are shown to be inconsistent. On the contrary, the mixture of stiffened gas and the modified barotropic EOS provide a coherent speed of sound.

Additional works are in progress to study cavitating flows with liquid hydrogen in cryogenic conditions.

\section{Appendix: Equilibrium speed of sound}

Starting from the usual thermodynamic relation :

$$
d e=T d s+\frac{P}{\rho^{2}} d \rho \quad \text { or } \quad d(\rho e)=\rho T d s+h d \rho
$$

And with the differential of $\rho e$ :

$$
d(\rho e)=\left(\frac{\partial \rho e}{\partial \rho}\right)_{P} d \rho+\left(\frac{\partial \rho e}{\partial P}\right)_{\rho} d P
$$

We can obtained the differential of the pressure $P$ :

$$
\left(\frac{\partial \rho e}{\partial P}\right)_{\rho} d P=\rho T d s+\left[h-\left(\frac{\partial \rho e}{\partial \rho}\right)_{P}\right] d \rho
$$

We deduce an expression of the speed of sound:

$$
c^{2}=\left(\frac{\partial P}{\partial \rho}\right)_{s}=\frac{h-\left(\frac{\partial \rho e}{\partial \rho}\right)_{P}}{\left(\frac{\partial \rho e}{\partial P}\right)_{\rho}}
$$

With the stiffened gas EOS, we have the following equalities:

$$
\left(\frac{\partial \rho e}{\partial \rho}\right)_{P}=\frac{\partial}{\partial \rho}\left[\alpha\left(\frac{P}{\gamma_{V}-1}+\rho_{V} q_{V}+\frac{\gamma_{V}}{\gamma_{V}-1} P_{\infty}^{V}\right)+(1-\alpha)\left(\frac{P}{\gamma_{L}-1}+\rho_{L} q_{L}+\frac{\gamma_{L}}{\gamma_{L}-1} P_{\infty}^{L}\right)\right]
$$




$$
=\frac{\rho_{L} h_{L}-\rho_{V} h_{V}}{\rho_{L}-\rho_{V}}
$$

And also :

$$
\begin{aligned}
\left(\frac{\partial \rho e}{\partial P}\right)_{\rho} & =\alpha\left(\frac{\partial \rho_{V} e_{V}}{\partial P}\right)_{\rho}+(1-\alpha)\left(\frac{\partial \rho_{L} e_{L}}{\partial P}\right)_{\rho}+\left(\rho_{V} h_{V}-\rho_{L} h_{L}\right)\left(\frac{\partial \alpha}{\partial P}\right)_{\rho} \\
& =\frac{1}{\gamma-1}-\frac{\rho_{L} h_{L}-\rho_{V} h_{V}}{\rho_{L}-\rho_{V}} \frac{d P}{d T}\left[\alpha\left(\frac{\partial \rho_{V}}{\partial T}\right)+(1-\alpha)\left(\frac{\partial \rho_{L}}{\partial T}\right)\right] \\
& =\frac{1}{\gamma-1}-\frac{\rho_{L} h_{L}-\rho_{V} h_{V}}{\rho_{L}-\rho_{V}} \frac{1}{T} \\
& \times\left[\alpha\left(\frac{1}{C v_{V}\left(\gamma_{V}-1\right)}-\rho_{V} \frac{d T}{d P}\right)+(1-\alpha)\left(\frac{1}{C v_{L}\left(\gamma_{L}-1\right)}-\rho_{L} \frac{d T}{d P}\right)\right]
\end{aligned}
$$

Moreover, according to the logarithmic relation between $P$ and $T$ (see [14]), the quantity $d P_{v a p} / d T(T)$ can be written as:

$$
\frac{d P}{d T}(T)=\frac{\frac{C}{T}-\frac{B}{T^{2}}}{\frac{1}{P+P_{\infty}^{V}}-\frac{D}{P+P_{\infty}^{L}}}
$$

where $B, C$ and $D$ are constant.

$$
B=\frac{q_{L}-q_{V}}{C p_{V}-C v_{V}} \quad: \quad C=\frac{C p_{V}-C p_{L}}{C p_{V}-C v_{V}} \quad ; \quad D=\frac{C p_{L}-C v_{L}}{C p_{V}-C v_{V}}
$$




\section{References}

[1] S. Clerc, Numerical simulation of the homogeneous equilibrium model for twophase flows. Journal of Computational Physics, 161(1):354-375 (2000).

[2] J.R. Edwards, R.K. Franklin, Low-Diffusion Flux Splitting methods for real fluid flows with phase transition. AIAA Journal, 38(9):1624-1633 (2000).

[3] J. Rolland, R. Fortes Patella, E. Goncalves, G. Boitel, S. Barre, Experiments and modelling of cavitating flows in Venturi, Part I: stable cavitation. Proceedings of sixth International Symposium on Cavitation CAV2006, Wageningen, The Netherlands, September 11-15 2006.

[4] R.F. Kunz, D.A. Boger, D.R. Stinebring, T.S. Chyczewski, J.W. Lindau, H.J. Gibeling, S. Venkateswaran, T.R. Govindan, A preconditioned Navier-Stokes method for two-phase flows with application to cavitation prediction. Computers Es Fluids, 29(8):849-875 (2000).

[5] A. Hosangadi, V. Ahuja, Numerical study of cavitation in cryogenic fluids. Journal of Fluids Engineering, 127(2):267-281 (2005).

[6] Y. Utturkar, J. Wu, G. Wang, W. Shyy, Recent progress in modelling of cryogenic cavitation for liquid rocket propulsion. Progress in Aerospace Sciences, 41:558$608(2005)$.

[7] X.B. Zhang, L.M. Qiu, Y. Gao, X.J. Zhang, Computational fluid dynamic study on cavitation in liquid nitrogen. Cryogenics, 48(9-10):432-438 (2008).

[8] E. Goncalves, R. Fortes Patella, Numerical Simulation of Cavitating Flows with Homogeneous Models, Computers \& Fluids, 38(9):1682-1696 (2009).

[9] E. Goncalves, R. Fortes Patella, Numerical study of cavitating flows with thermodynamic effect, Computers \& Fluids, 39(1):99-113 (2010).

[10] R. Menikoff, and B.J. Plohr, The Riemann problem for fluid flow of real materials, Reviews of Modern Physics, 61(1):75-130 (1989). 
[11] V. Perrier, The Chapman-Jouguet closure for the Riemann problem with vaporization, SIAM Journal On Applied Mathematics, 68(5): 1333-1359 (2008).

[12] G. Wallis, One-dimensional two-phase flow. New York, McGraw-Hill, 1967.

[13] F. Petitpas, J. Massoni, R. Saurel, E. Lapebie, and L. Munier, Diffuse interface model for high speed cavitating underwater systems. Int. Journal of Multiphase Flow, 35:747-759 (2009).

[14] R. Saurel, F. Petitpas, and R. Abgrall, Modelling phase transition in metastable liquids: application to cavitating and flashnig flows. Journal of Fluid Mechanics, 607:313-350 (2008).

[15] Y. Delannoy, J.-L. Kueny, Two phase flow approach in unsteady cavitation modelling, in Cavitation and Multiphase Flow Forum, ASME-FED, 98:153-158 (1990). 
Table 1

Parameters of the stiffened gas EOS, freon R-114, $T_{r e f}=293 \mathrm{~K}$.

\begin{tabular}{lllllll}
\hline & $\gamma$ & $P_{\infty}(\mathrm{Pa})$ & $\mathrm{q}(\mathrm{J} / \mathrm{kg})$ & $C_{p}(\mathrm{~J} / \mathrm{K} . \mathrm{kg})$ & $\rho\left(\mathrm{kg} / \mathrm{m}^{3}\right)$ & $h(\mathrm{~kJ} / \mathrm{kg})$ \\
\hline liquid & 1.1 & $3.83510^{7}$ & $-0.6910^{5}$ & 984 & 1470.3 & 219300 \\
vapour & 1.07 & 0 & $0.14210^{6}$ & 700 & 13.52 & 349340 \\
\hline
\end{tabular}


Table 2

$\underline{c_{\text {baro }} \text { values and convexity domain, freon R-114. }}$

\begin{tabular}{llllll}
$T_{\text {ref }}(\mathrm{K})$ & $\rho_{L}\left(\mathrm{~kg} / \mathrm{m}^{3}\right)$ & $\rho_{V}\left(\mathrm{~kg} / \mathrm{m}^{3}\right)$ & $c_{\text {baro }}(\mathrm{m} / \mathrm{s})$ & $\Delta P(\mathrm{~Pa})$ & convexity domain \\
\hline 293 & 1470.6 & 13.52 & 2.61 & 7794 & $\alpha<0.9$ \\
293 & 1470.4 & 13.52 & 1.48 & 2551 & $\alpha<0.8$ \\
293 & 1470.33 & 13.52 & 0.74 & 638 & $\alpha<0.7$ \\
\hline
\end{tabular}




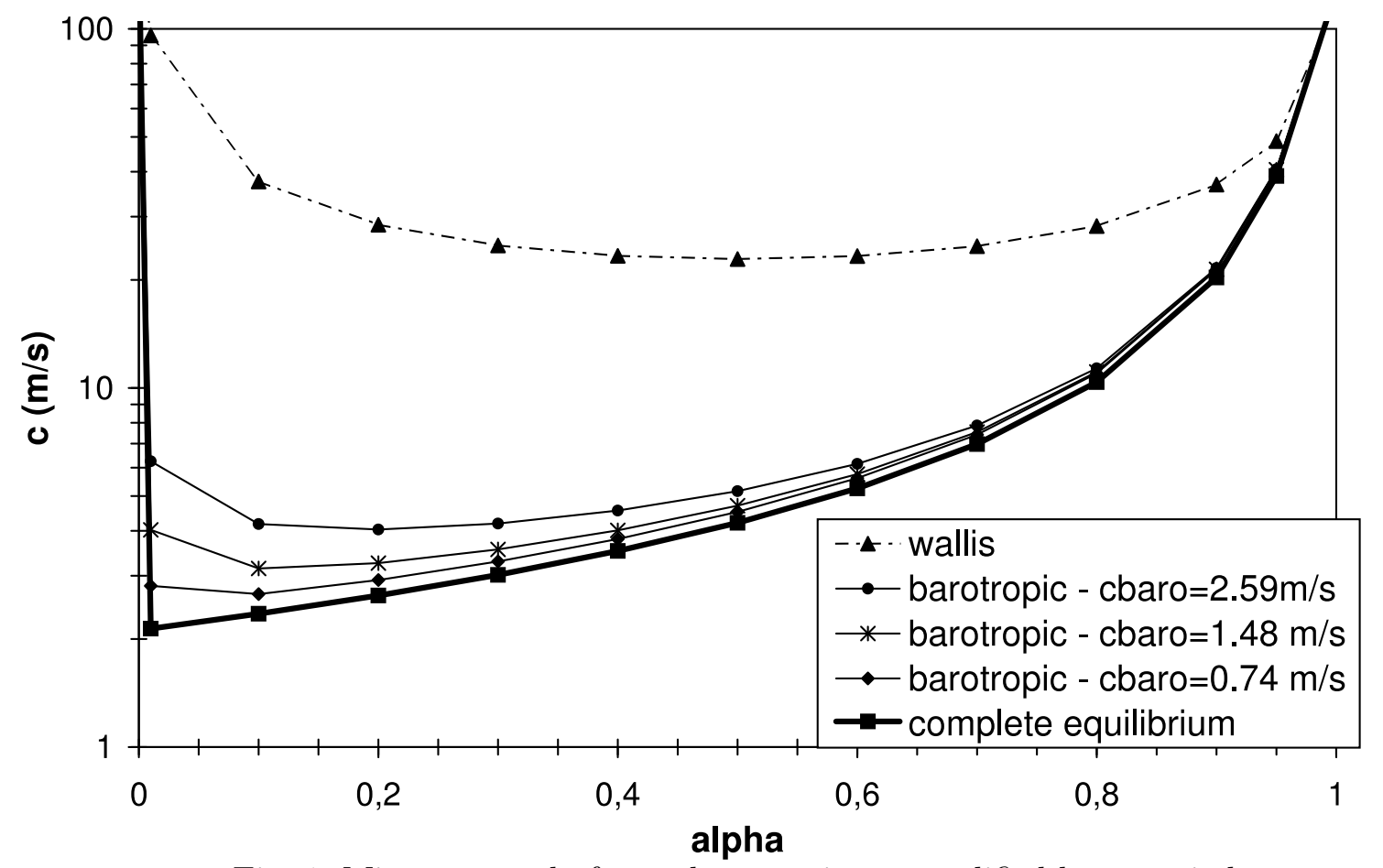

Fig. 1. Mixture speed of sound comparisons, modified barotropic law 


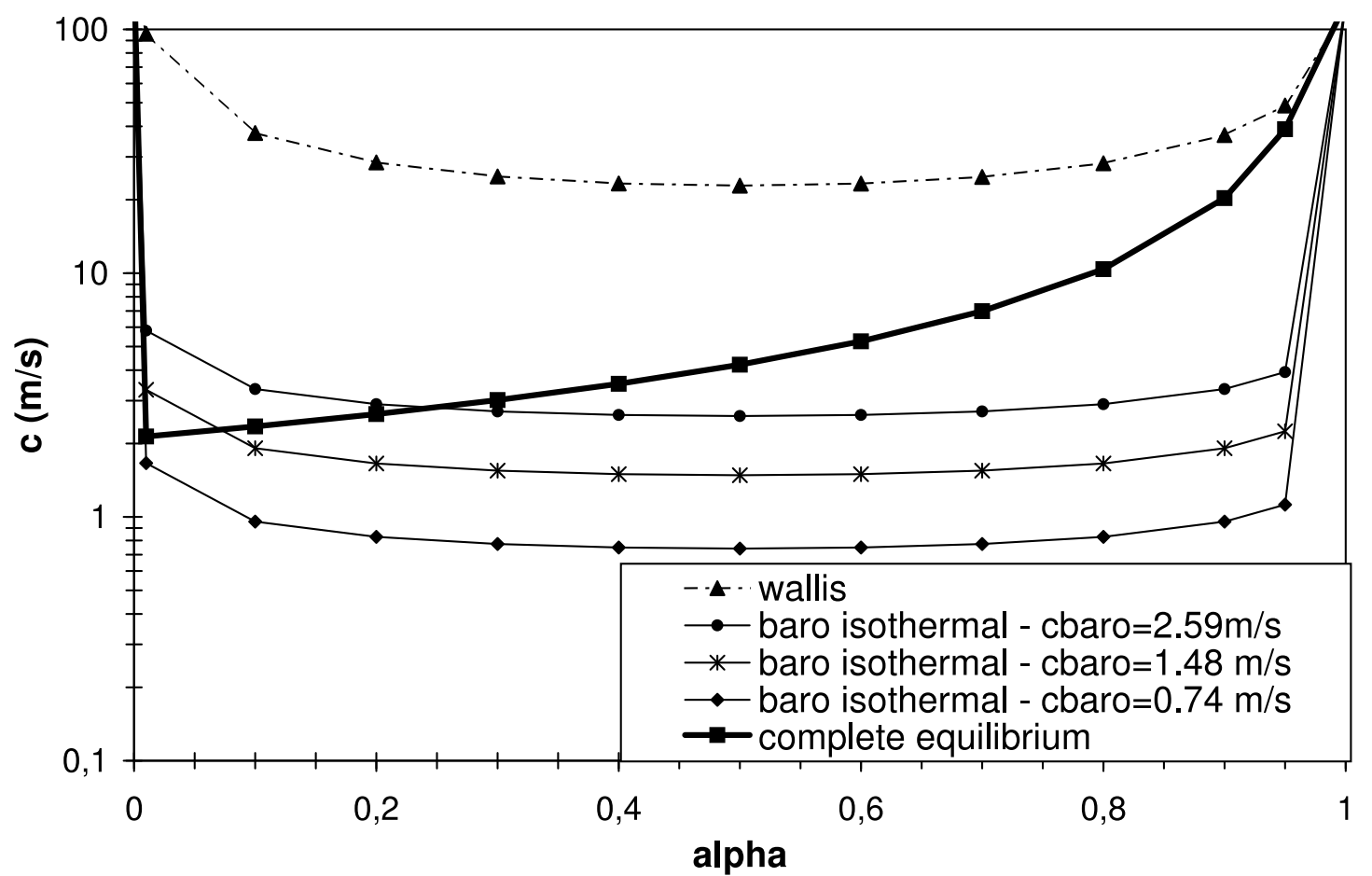

Fig. 2. Mixture speed of sound comparisons, isothermal barotropic law 


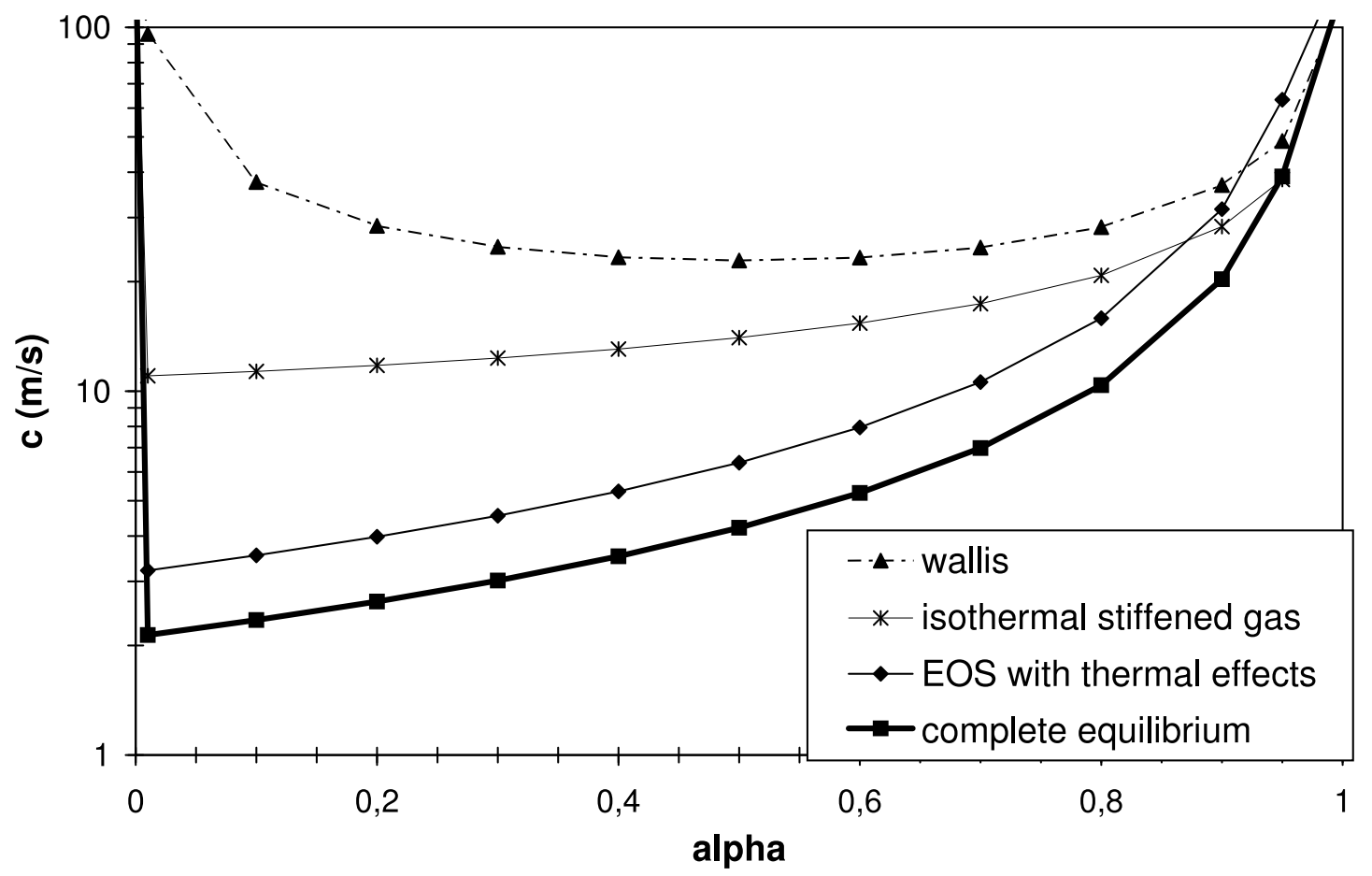

Fig. 3. Mixture speed of sound comparisons, mixture of stiffened gas 